\title{
Prédiction de la cavitation liée à l'enroulement en extrémité d'un hydrofoil de forme en plan elliptique
}

\author{
par Ph. Dupont, R. Hirschi \\ EPFL-LMH, Lausanne
}

\author{
J.-Y. Billard
}

EN-LH, Brest

\section{I 口 INTRODUCTION}

La cavitation est un facteur limitatif pour le domaine d'utilisation des propulseurs marins, en particulier pour les bâtiments submersibles, puisqu'elle nuit à leur discrétion. Parmi les différentes formes sous lesquelles la cavitation peut apparaître, la plus précoce est la cavitation de tourbillon marginal, liée à l'enroulement en extrémité des plans porteurs et notamment des pales des hélices. Une prédiction précise, par un calcul d'écoulement, des pressions liées à ce phénomène d'enroulement permettrait à l'ingénieur, dans la phase de conception du propulseur, d'en optimiser le dessin.

Le but de cet article est de vérifier la possibilité de cette prédiction par l'utilisation de codes de calcul disponibles sur le marché et représentant les grandes tendances des développements actuels. Les progrès récents tant au niveau des algorithmes que des performances des ordinateurs permettent d'envisager l'utilisation de codes de calcul prenant en compte la nature à la fois visqueuse et turbulente des écoulements autour de formes complexes. Si les résultats obtenus à l'aide des codes Navier-Stokes sont très satisfaisants pour des plans d'envergure infinie - bidimensionnels, ce n'est pas encore le cas pour des ailes d'envergure finie - tridimensionnelles. Pour celles-ci, l'écoulement de contournement en leur extrémité est à l'origine d'une mise en rotation intense : le tourbillon marginal. Les études effectuées à ce jour semblent indiquer que les modèles de turbulence classiques ne sont pas à même de prendre en compte de tels écoulements [3]. D'autre part, cette mise en rotation, s'établissant à une échelle géométrique très inférieure à celle de l'aile, exige un grand raffinement du maillage en extrémité de l'aile et sur la trajectoire du tourbillon. Récemment, plusieurs auteurs se sont intéressés à ce problème de modélisation (Dacles Mariani [4], Deniset [6], Dupont [5]). A l'analyse de ces travaux, il apparaît que les principales difficultés associées à l'utilisation de ces codes sont : la modélisation de la turbulence, la discrétisation spatiale dans la région du tourbillon et l'application de la condition limite sur la frontière aval du domaine de calcul.

Cet article présente les tentatives de calcul de l'écoulement tridimensionnel en extrémité d'une aile effectuées à l'aide de trois codes utilisant différentes méthodes de résolution. Les résultats obtenus sont comparés à des mesures du champ de vitesse effectuées en veine d'essai. Une attention particulière est portée à l'enroulement dans le sillage proche de l'extrémité de l'aile et aux vitesses tangentielles associées au tourbillon.
La comparaison des résultats obtenus montre une influence très importante du choix du solveur, du modèle de turbulence, ainsi que de la discrétisation spatiale sur le résultat du calcul. Afin d'analyser l'importance de ces paramètres, un cas test, simplifiant le problème du calcul de la diffusion d'un tourbillon axial, est présenté. Il correspond à la propagation d'un tourbillon de type Oseen axisymétrique dans un tube à parois solides. Ce calcul est réalisé en utilisant différentes combinaisons de densités de mailles, de modèles de turbulence et de solveurs.

\section{II $\square$ MONTAGE EXPERIMENTAL}

Un profil de forme en plan elliptique de section NACA 16020 , d'une corde maximale à l'emplanture de $80 \mathrm{~mm}$ et d'une envergure de $120 \mathrm{~mm}$ a été testé dans la veine d'essai du tunnel de cavitation du Laboratoire d'Hydrodynamique de l'Ecole navale de Brest. Ce profil est monté horizontalement dans une veine de section carrée de $192 \mathrm{~mm}$ de côté et de $1 \mathrm{~m}$ de long.

Les mesures du champ de vitesse dans le sillage proche de l'extrémité de la pale ont été effectuées par vélocimétrie laserDoppler. Un système LDA à deux composantes utilisé en mode par rétrodiffusion donne l'accès aux composantes axiales et verticales de la vitesse. Le traitement du signal Doppler est fait à l'aide de deux analyseurs de spectre (Dantec BSA). Les profils de vitesses, effectués avec un pas de $50 \mu \mathrm{m}$, correspondent en

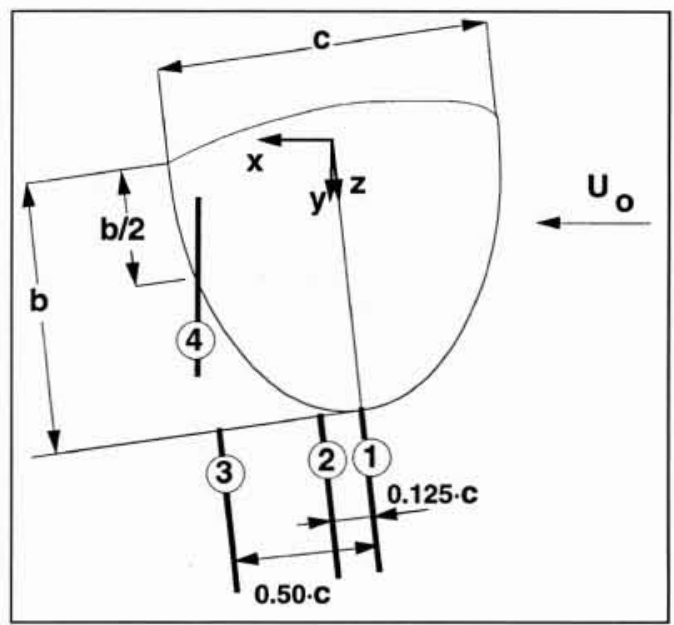

1. Position des profils de vitesse. 
chaque point de mesure, à une moyenne basée sur 1500 mesures instantanées. Le système de mesure et sa précision sont largement décrits dans [1].

Les résultats présentés ci-après ont été obtenus pour une incidence de $10^{\circ}$ et une vitesse d'écoulement en entrée de veine de $15 \mathrm{~ms}^{-1}$, correspondant à un nombre de Reynolds, basé sur la corde du profil à son emplanture, de $1,2.10^{6}$. Le choix d'une incidence de $10^{\circ}$ a été motivé par l'intérêt de comparer des résultats numériques et expérimentaux pour un écoulement présentant à la fois un décollement et un enroulement d'extrémité.

Trois profils de vitesse verticale effectués à proximité de l'extrémité du profil sont présentés et comparés aux résultats numériques, ainsi qu'un profil de vitesse axiale effectué à la verticale du bord de fuite du profil à mi-envergure, comme le montre la Figure 1.

Les trois premiers axes de sondage permettent d'évaluer la modélisation de l'enroulement dans le sillage proche du profil en son extrémité. Ils sont dirigés selon l'envergure du profil (axe z) et situés respectivement en son extrémité (1), à 0,125 fois (2) et à 0,5 fois (3) la corde à l'emplanture du profil dans son sillage. La position verticale des sondages est choisie afin qu'elle coupe la trajectoire du tourbillon d'extrémité.

Un quatrième axe de sondage, dirigé selon l'axe y et situé à la verticale du bord de fuite du profil à mi-envergure (4), a également été utilisé pour la comparaison entre la mesure et le calcul. Il permet d'estimer la qualité de la représentation de la couchelimite, et notamment du décollement qui influence notablement la formation et la convection du tourbillon d'extrémité.

\section{III घ MODÉLISATION NUMÉRIQUE}

Trois codes de calcul d'écoulement ont été utilisés, résolvant l'un l'équation d'Euler (Euler-IMHEF), et les deux autres l'équation de Navier-Stokes. Pour ces derniers, la différence principale réside dans leur méthode de résolution, l'un utilisant la méthode des volumes finis (TascFlow ${ }^{\mathrm{TM}}$ ), l'autre la méthode des éléments finis (Fidap ${ }^{\mathrm{TM}}$ ). Essentiellement deux modèles de turbulence ont été utilisés pour cette étude : un modèle 0-équation de type "longueur de mélange" et un modèle 2-équations de type $\mathrm{k}-\varepsilon$, avec, dans le cas de Fidap ${ }^{\mathrm{TM}}$, une correction pour tenir compte des courbures des lignes de courant.

\section{- 3.1 Profil elliptique en veine}

L'écoulement autour d'un profil de forme en plan elliptique sans dévers monté en veine d'essai est modélisé dans des conditions identiques à l'expérience. Afin d'être en homologie la plus complète avec l'expérience, les parois verticales et horizontales de la veine ont été modélisées.

\subsubsection{Maillage du plan porteur en veine d'essai}

Une des difficultés principales du problème de la modélisation de l'écoulement autour d'un plan porteur à envergure finie est la génération d'un maillage respectant au mieux la géométrie de son extrémité. Cette difficulté est accrue par le fait que certains codes de calcul nécessitent la génération d'un maillage structuré, ce qui implique, en extrémité du plan porteur, soit une dégénérescence des éléments, soit une corde non nulle.

Les maillages utilisés pour ces calculs ont été réalisés en tenant compte de la spécificité des différents codes. Pour les codes volumes finis (Euler et TascFlow ${ }^{\mathrm{TM}}$ ), un maillage structuré monobloc de type $\mathrm{C}-\mathrm{H}$ formé d'éléments hexaédriques a été réalisé (Figure 2a). Pour le calcul TascFlow ${ }^{\mathrm{TM}}$, le maillage monobloc, utilisé dans un premier temps, a été raffiné sur une sous-grille dans les zones d'intérêt (Figure $2 b$ ).

Ce raffinement est réalisé en multipliant par deux le nombre des cellules dans les directions perpendiculaires à l'écoulement principal. Le nombre total de nœuds pour le premier maillage est d'environ 90000 , et d'un peu plus de 200000 pour le maillage raffiné. La face d'entrée du domaine de calcul est placée à deux cordes à l'amont du profil, et la face de sortie à cinq cordes en aval.

Pour le code de calcul éléments finis (Fidap ${ }^{\mathrm{TM}}$ ), un maillage multibloc a été généré. La structure logique de ce maillage est présentée sur la Figure 3a. L'aube est formée de cinq faces logiques, raccordées à cinq blocs correspondant aux volumes

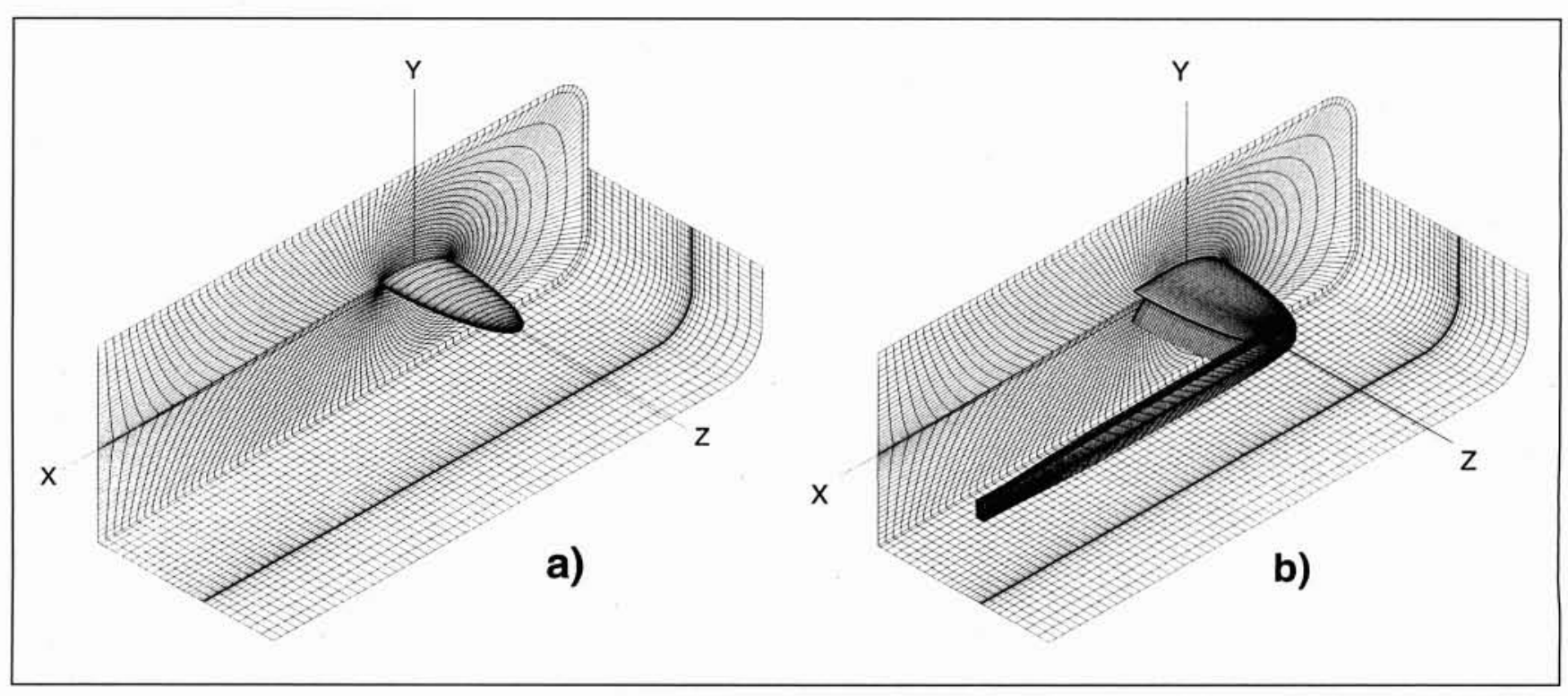

2. Maillage monobloc C-H (Euler \& TascFlow ${ }^{\mathrm{TM}}$ ) (a) et raffiné en extrémité (TascFlow ${ }^{\mathrm{TM}}$ raffiné) (b). 


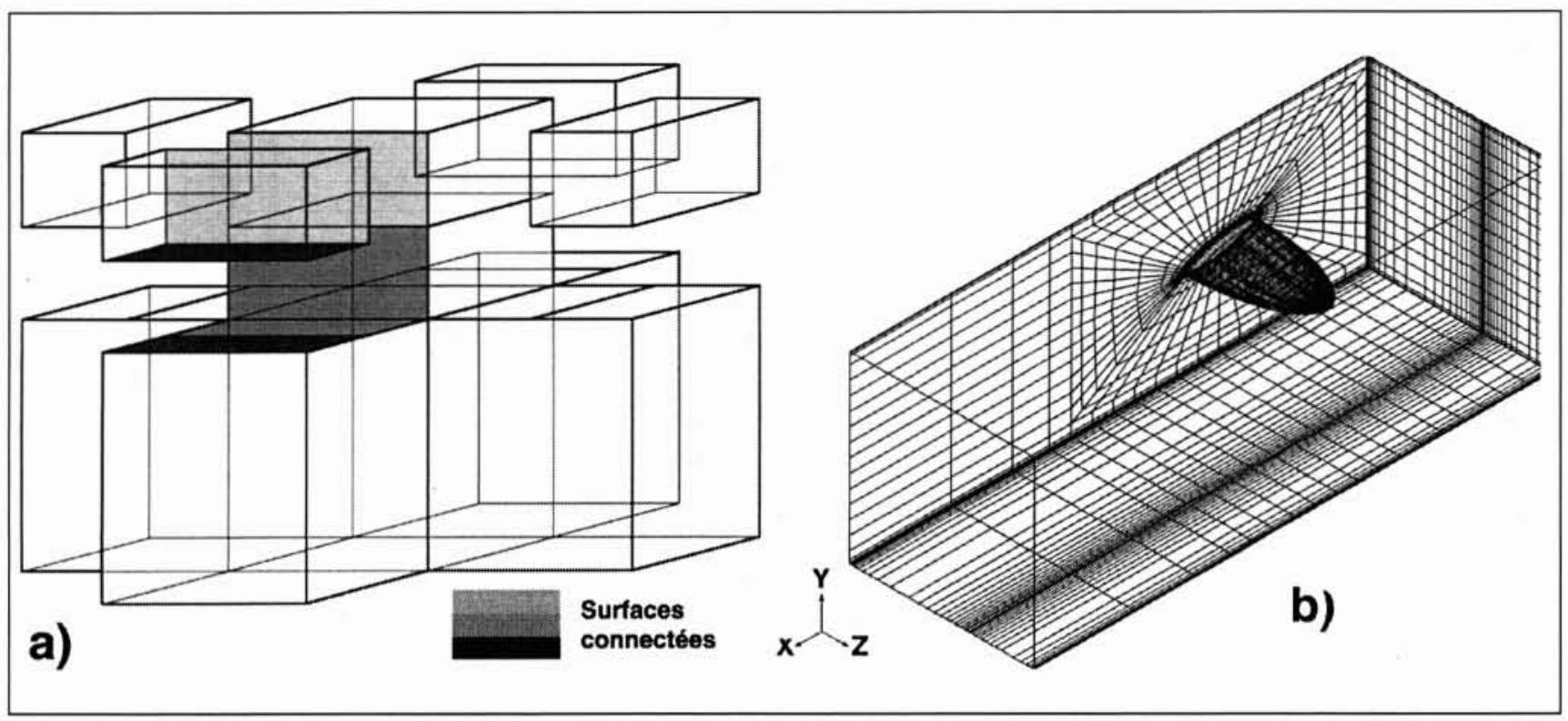

3. Structure logique (a) et maillage multibloc (FIDAPTM) (b).

amont, aval, extrados, intrados et supérieur du profil. Les éléments utilisés sont de type hexaédriques. Des nappes de transition, formées d'éléments tétraédriques, ont été utilisées pour densifier le maillage autour du plan porteur. Le nombre total de nœuds pour ce maillage est d'environ 70000 . Comme pour les maillages précédents, les faces d'entrée et de sortie sont situées respectivement à deux cordes à l'amont et à cinq cordes à l'aval du profil.

\subsubsection{Conditions aux frontières du domaine de calcul et conditions de calcul}

Pour le code Euler, une vitesse débitante uniforme et une vitesse transversale nulle sont imposées sur la face d'entrée du maillage. Des conditions d'imperméabilité (vitesse normale nulle) sur les faces solides que sont les parois de la veine et celles du profil sont prescrites. En sortie, une condition de pression constante a été définie.

Pour le code Navier-Stokes volumes finis, une vitesse débitante constante et une vitesse transversale nulle sont également spécifiées en entrée du domaine de calcul. Une condition d'adhérence est imposée sur les parois de la veine d'essai, ainsi qu'aux parois du profil. En sortie du domaine de calcul, une condition de pression constante est fixée. Un modèle de turbulence $k-\varepsilon$ standard est utilisé. Les conditions d'entrée pour ce calcul de la turbulence sont une intensité de $5 \%$ et une longueur caractéristique égale à $1 / 50$ de la corde du profil.

Pour le code Navier-Stokes éléments finis, les mêmes conditions d'entrée sont imposées. Le modèle de turbulence utilisé est également un modèle $\mathrm{k}-\varepsilon$, mais étendu avec la prise en compte des courbures des lignes de courant. Les parois de la veine et du profil sont également considérées comme solides avec une vitesse nulle. Afin de modéliser l'écoulement à bas nombre de Reynolds à proximité de ces surfaces solides, une loi de paroi est utilisée. Un schéma de calcul découplé (segr.) est utilisé, étant le seul à permettre de résoudre, par la méthode des éléments finis, un problème de cette taille.

\section{- 3.2 Comparaison des résultats numériques et expérimentaux}

La comparaison du résultat de ces trois codes de calcul avec l'expérience a été faite principalement sur la base des relevés des composantes tangentielles et axiales de la vitesse sur les quatre axes de sondage indiqués sur la Figure 1. Comme lors de la mesure, la position verticale des sondages est choisie afin qu'elle coupe la trajectoire du tourbillon d'extrémité.

Sur les trois premiers axes de sondage, la vitesse verticale relative Uy/Uo calculée a été comparée à la mesure par vélocimétrie laser-Doppler. Ces résultats sont présentés pour la station $\mathrm{x} / \mathrm{C}=0$ (1) sur la Figure 4, pour la station $\mathrm{x} / \mathrm{C}=0,125$ (2) sur la Figure 5 et pour la station $\mathrm{x} / \mathrm{C}=0,5$ (3) sur la Figure 6 .

Sur le dernier axe de sondage (4), la vitesse axiale relative Ux/Uo calculée a été comparée à la mesure. Cette comparaison est donnée sur la Figure 7.

Le rapport entre les maxima de vitesse tangentielle calculés et mesurés aux diverses stations de sondage est synthétisé sur le Tableau 1.

Tableau 1: Rapport entre les vitesses tangentielles maximum calculées et mesurées pour les trois sections en extrémité du profil.

\begin{tabular}{|l|c|c|c|}
\hline $\begin{array}{c}\text { Station de sondage } \\
\text { Code }\end{array}$ & $\mathrm{x} / \mathrm{C}=0,0$ & $\mathrm{x} / \mathrm{C}=0,125$ & $\mathrm{x} / \mathrm{C}=0,50$ \\
\hline Euler & 0,77 & 0,42 & 0,33 \\
\hline Fidap $^{\mathrm{TM}}$ & 1,46 & 0,60 & 0,44 \\
\hline TascFlow $^{\mathrm{TM}}$ & 1,30 & 0,59 & 0,38 \\
\hline TascFlow $^{\mathrm{TM}}$ raffiné & 1,68 & 0,80 & 0,49 \\
\hline
\end{tabular}




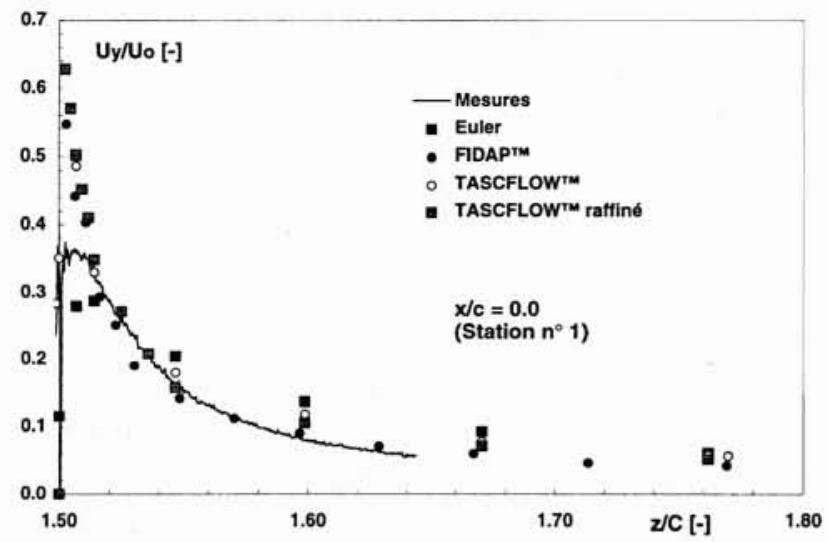

4. Comparaison des profils de vitesse verticale relative $U_{y} / U_{o}$ mesurés et calculés en extrémité du plan porteur.

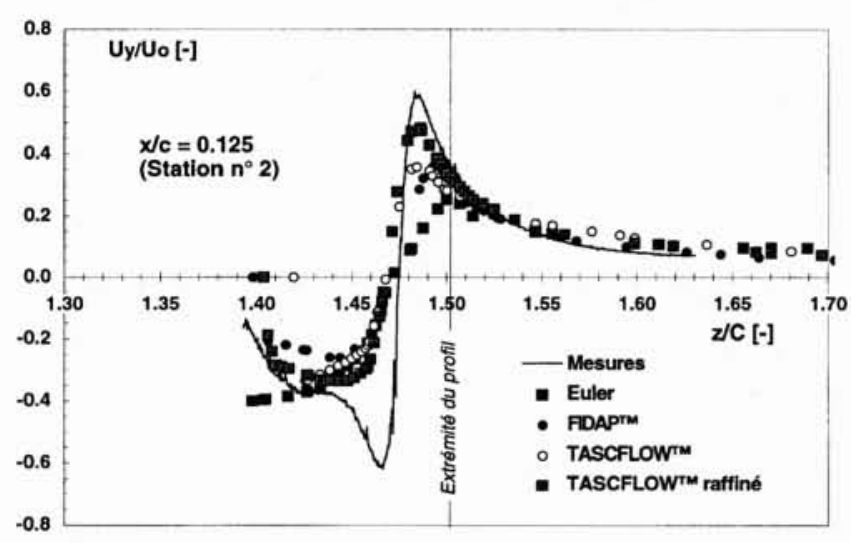

6. Comparaison des profils de vitesse verticale relative $U_{y} / U_{o}$ mesurés et calculés pour la station $\mathbf{x} / \mathbf{C}=\mathbf{0 , 5}$.

\subsection{Cas test}

Afin d'étudier plus spécifiquement le problème de la diffusion d'un tourbillon axial, un cas test est résolu à l'aide du code Fidap ${ }^{\mathrm{TM}}$. Ce code a été choisi pour cette étude en raison de ses grandes possibilités de paramétrisation. Ce cas test correspond à la propagation d'un tourbillon dans un tube. Il est traité en coordonnées cylindriques $\left(\mathrm{U}_{r}, \mathrm{U}_{z}, \mathrm{U}_{\vartheta}\right)$ dans un plan méridien $(\mathrm{r}, \mathrm{z})$ en supposant que les dérivées tangentielles $\partial / \partial \theta$ sont nulles. En entrée du tube, une vitesse tangentielle correspondant à un tourbillon d'Oseen et une vitesse axiale constante sont imposées. Le tourbillon a un cœur visqueux d'un rayon égal à un centième du rayon du tube $\mathrm{R}$ et une vitesse tangentielle maximum égale à 0,12 fois la vitesse axiale. Le nombre de Reynolds, basé sur le rayon du tube et sur la vitesse axiale en entrée, est égal à $10^{6}$. Des conditions de vitesse radiale nulle sur l'axe et d'adhérence à la paroi du tube sont également imposées. En sortie du domaine de calcul, située à 10R à l'aval de la face d'entrée, une condition de contraintes nulles est fixée.

Un maillage structuré de 161 nœuds dans la direction axiale et de 69 nœuds dans la direction radiale est utilisé pour étudier l'influence du solveur et du modèle de turbulence. L'influence de la densité du maillage est également testée en utilisant deux autres maillage de densité moindre.

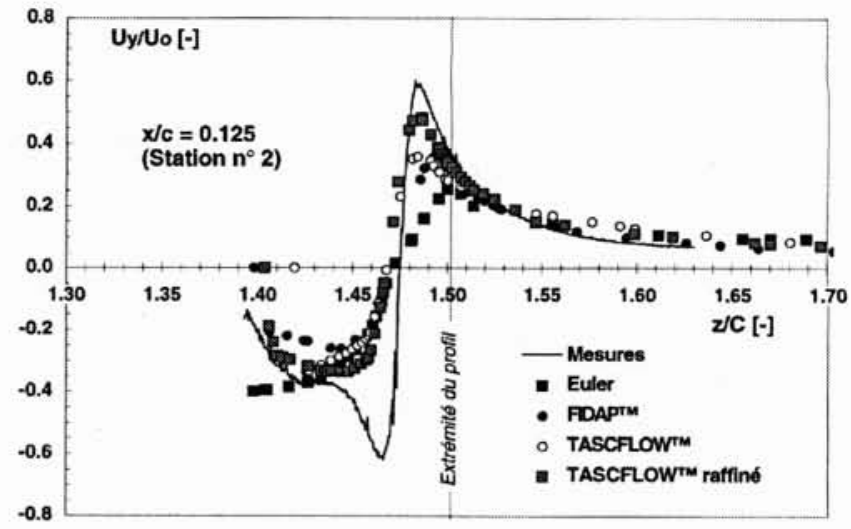

5. Comparaison des profils de vitesse verticale relative $U_{y} / U_{o}$ mesurés et calculés pour la station $\mathrm{x} / \mathrm{C}=\mathbf{0 , 1 2 5}$.

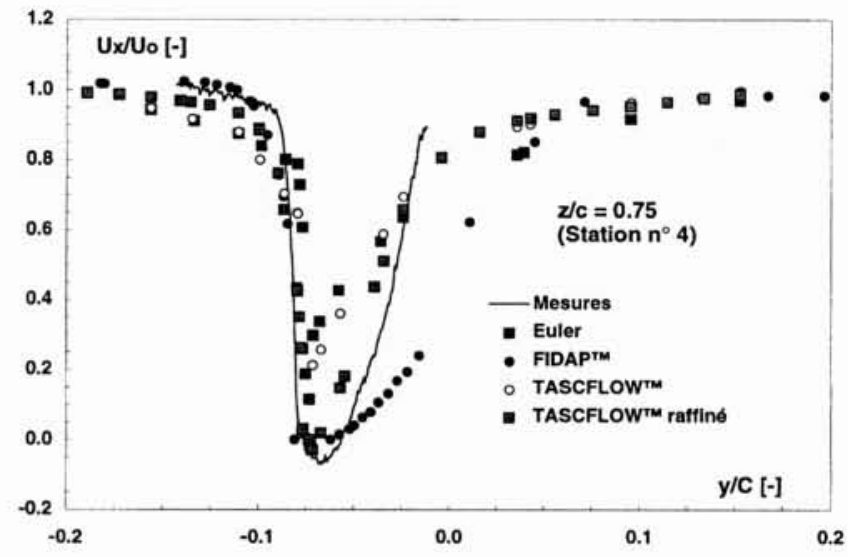

7. Comparaison des profils de vitesse axiale relative $U_{x} / U_{0}$ mesurés et calculés à la verticale du bord de fuite à mienvergure.

\subsubsection{Influence du solveur}

Deux solveurs sont utilisés : un schéma direct utilisant la méthode dite de Picard ou d'approximations successives (ciaprès désigné par l'abréviation s.s.) et un schéma découplé par bloc (ci-après désigné par l'abréviation segr.) avec une décomposition de type gradient-conjugué. Ce second schéma, réputé moins précis, mais beaucoup moins gourmand en ressources de calcul, est le seul possible pour traiter des cas de calcul tridimensionnel de grandes dimensions.

L'évolution de la vitesse tangentielle maximum le long de l'axe du tube est donnée sur la Figure 8 pour ces deux solveurs et les modèles de turbulence k- $\varepsilon$ et longueur de mélange. Sur la Figure 9, l'évolution du rayon du cœur du tourbillon est donnée en fonction de la position axiale le long du tube. Les "accidents" dans cette évolution sont dus à la discrétisation radiale du maillage.

\subsubsection{Influence du modèle de turbulence}

Pour en étudier l'influence, deux modèles de turbulence sont utilisés. Ces deux modèles ont été testés avec le schéma découplé (segr.) et un maillage fin. 


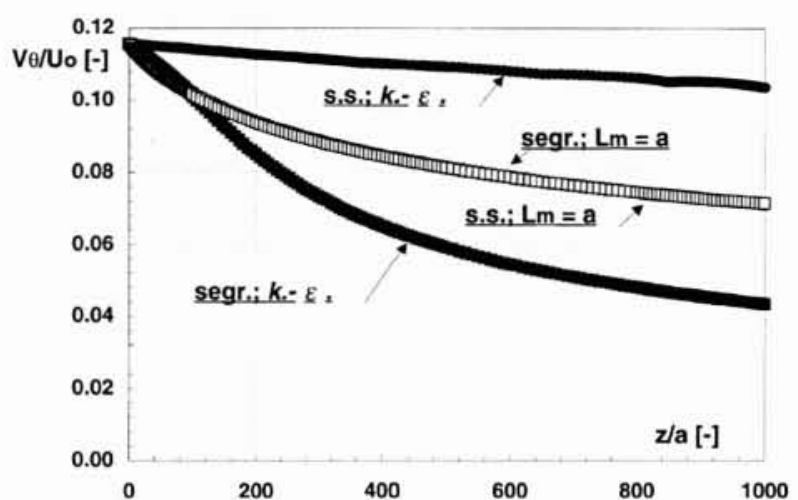

8. Influence du solveur sur l'évolution de la vitesse tangentielle maximum calculée le long du tube.

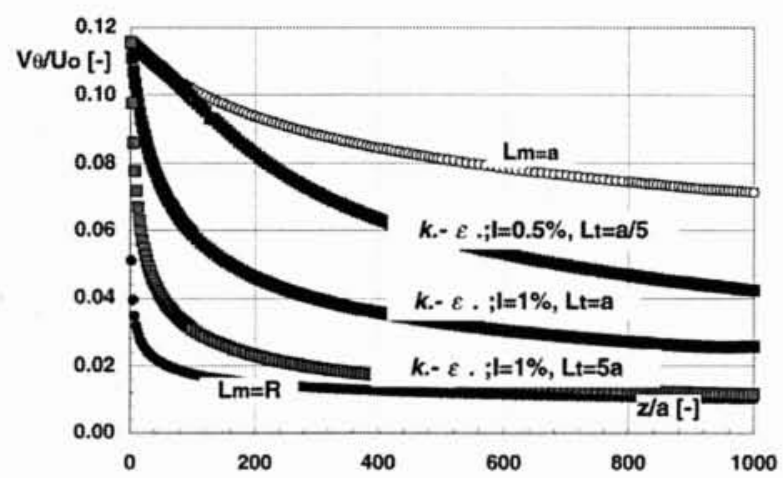

10. Influence du modèle de turbulence sur l'évolution de la vitesse tangentielle maximum calculée le long du tube.

Le premier modèle est du type longueur de mélange classique. L'échelle caractéristique de longueur des macrostructures de la turbulence $\mathrm{L}_{\mathrm{m}}$ est imposée. Deux valeurs extrêmes sont choisies, la première correspondant au rayon du tube $\left(\mathrm{L}_{\mathrm{m}}=\mathrm{R}\right)$, la deuxième au diamètre du cocur visqueux du tourbillon $\left(\mathrm{L}_{\mathrm{m}}=\mathrm{a}\right)$. Le second modèle est du type $k-\varepsilon$ classique avec une correction pour prendre en compte les courbures des lignes de courant. Les conditions à l'entrée du domaine de calcul pour les valeurs de l'énergie cinétique de la turbulence $\mathrm{k}$ ainsi que pour la dissipation $\varepsilon$ sont imposées par l'intermédiaire d'une intensité de la turbulence amont et d'une échelle de longueur de la turbulence. Trois couples de valeurs ont été imposés, avec une intensité de la turbulence égale à 0,5 et $1 \%$ de l'énergie cinétique amont et une échelle de la turbulence, relative au coeur visqueux du tourbillon a, égale à a $/ 5$, a et $5 \mathrm{a}$.

Des extensions du modèle de turbulence $k-\varepsilon$ classique, l'une du type $\mathrm{k}-\omega$ ou l'autre à viscosité anisotropique, ont également été utilisées. $\mathrm{N}$ 'ayant pas montré de résultats significativement différents par rapport au modèle de base, elles ne sont pas reportées ici.

Les résultats pour les diverses paramétrisations de ces deux modèles de turbulence sont donnés sous la forme de l'évolution de la vitesse tangentielle maximum le long du tube, ainsi que l'évolution du cœur visqueux du tourbillon respectivement sur la Figure 10 et la Figure 11.

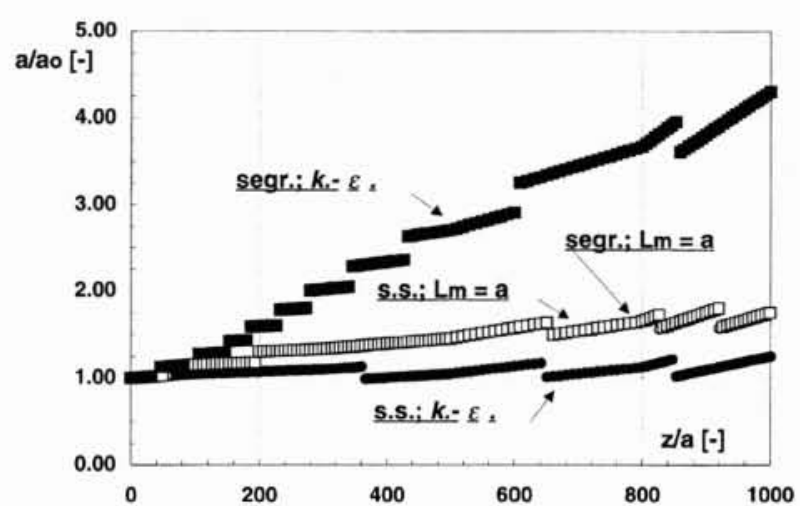

9. Influence du solveur sur l'évolution du rayon du cœur du tourbillon calculé le long du tube.

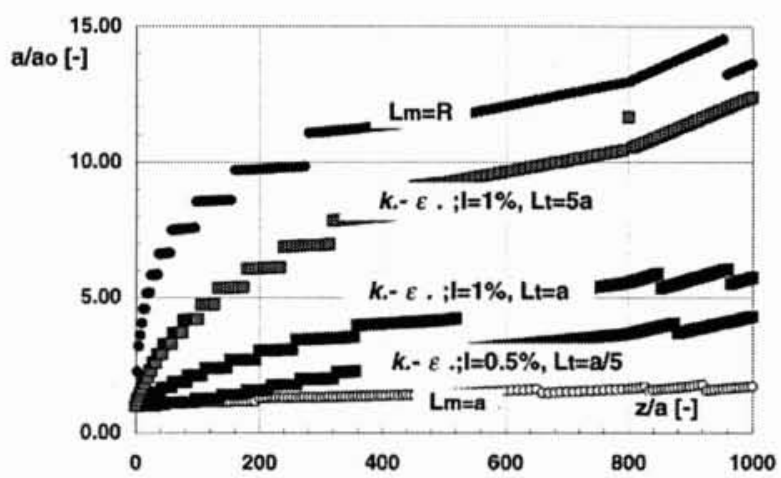

11. Influence du modèle de turbulence sur l'évolution du rayon du cœur visqueux du tourbillon calculé le long du tube.

\subsubsection{Influence du maillage}

Enfin, pour étudier l'influence de la discrétisation spatiale du domaine de calcul sur la propagation axiale d'un tourbillon, trois maillages sont utilisés. Ces trois maillages, présentés sur la Figure 12, sont formés de 161 points dans la direction axiale et 69 points dans direction radiale pour le plus dense, de $81 \times 35$ nœuds pour la densité intermédiaire, et de 81 x 13 nœuds pour le maillage grossier. Pour ces trois densités, le nombre de cellules à l'intérieur du cœur visqueux du tourbillon est respectivement de 20,10 et 4.

L'évolution du maximum de la vitesse tangentielle (Figure 13), ainsi que celle du rayon du cœur visqueux du tourbillon (Figure 14), est suivie pour ces trois maillages en utilisant un schéma découplé (segr.) et un modèle de longueur de mélange.

\section{IV $\square$ DISCUSSION}

\subsection{Analyse du cas de l'aile elliptique}

Les résultats obtenus dans le cas de l'aile elliptique montrent que tous les codes visqueux surestiment le coutournement en extrémité (Profil $\mathrm{x} / \mathrm{C}=0$.). Cette situation est probablement due à la géométrie du maillage correspondant à cette extrémité du 


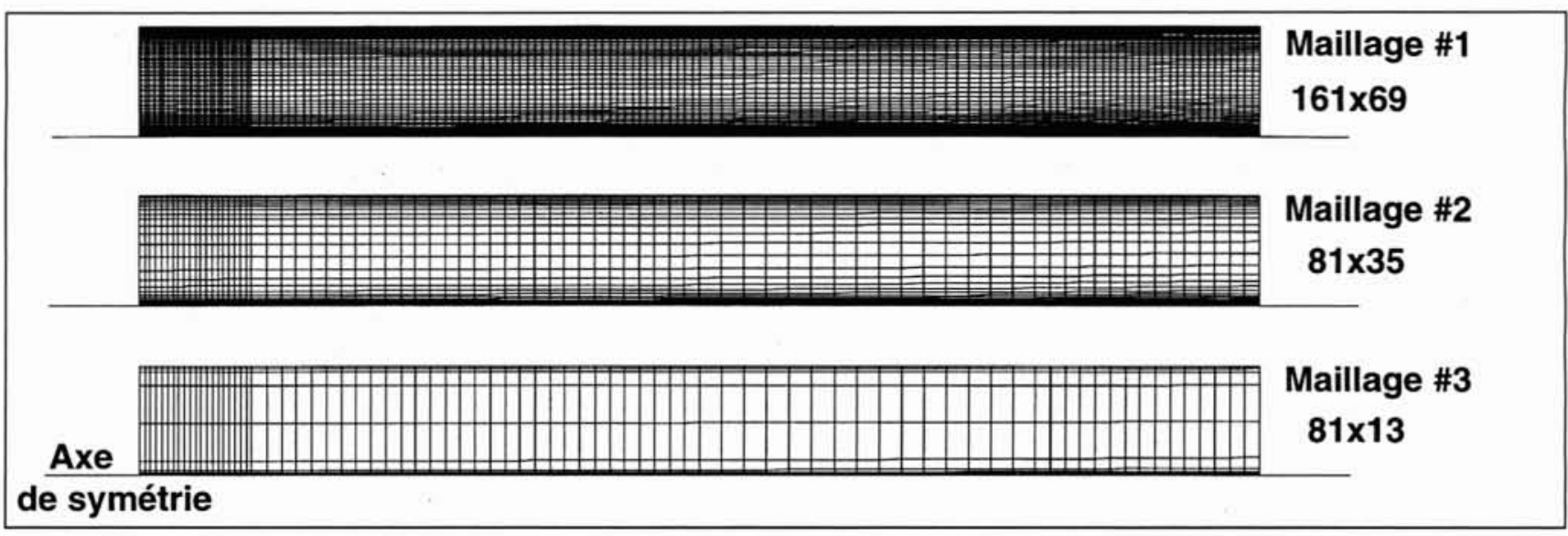

12. Maillage du cas test axisymétrique avec différentes densités.

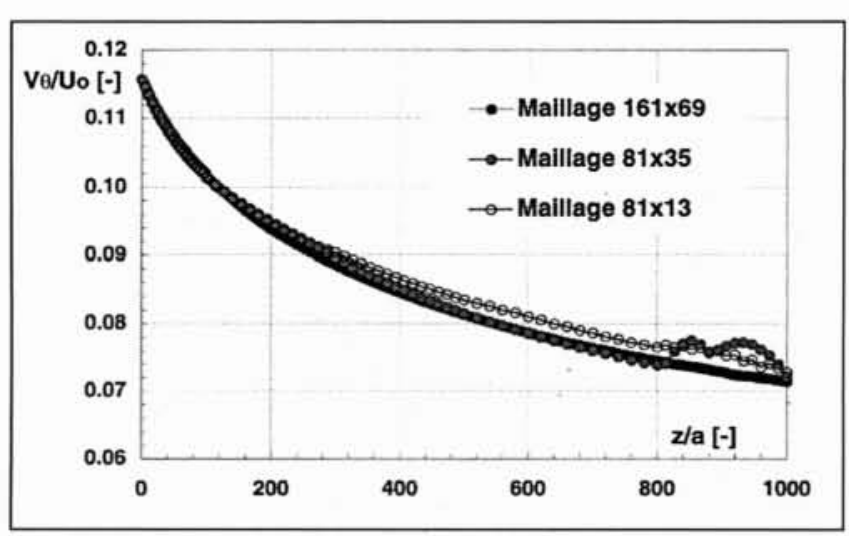

13. Influence du maillage sur l'évolution de la vitesse tangentielle maximum calculée le long du tube.

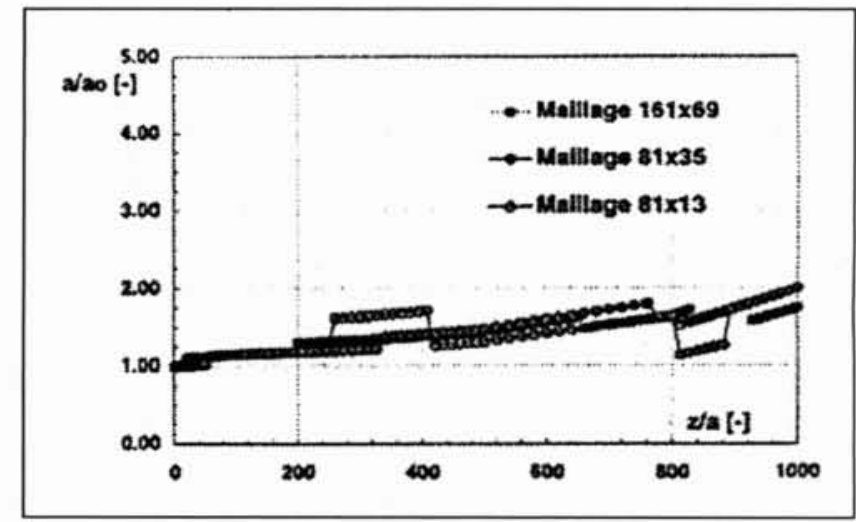

14. Influence du maillage sur l'évolution du rayon du cœur visqueux du tourbillon calculé le long du tube. profil. En effet, alors que le profil réel est une surface gauche, la modélisation qui en est faite correspond à une surface réglée s'appuyant sur des coupes imposées. Cette manière de procéder induit, dans les régions à fortes courbures comme l'extrémité du profil, des facettes avec des angles vifs. C'est donc probablement l'angle en extrémité du profil qui induit cette surestimation des vitesses de contournement. Ces défauts de géométrie ont très certainement également des conséquences sur la formation de la couche-limite et du sillage du profil dans la région de l'extrémité du profil, ainsi que sur la trajectoire calculée du tourbillon. Le code non visqueux Euler semble quant à lui sous-estimer les vitesses verticales sur cette section, comme d'ailleurs sur les autres axes de mesure. La zone du tourbillon en rotation solide est quant à elle surévaluée par le code Euler.

Les codes Navier-Stokes volumes finis et éléments finis donnent un résultat quasiment identique sur tous les axes de sondages pour un maillage de même densité. Pour une densité supérieure, la prédiction de la vitesse tangentielle maximum est améliorée de façon significative dans la région du tourbillon hors sillage. Dans le sillage du profil, la vitesse tangentielle liée au tourbillon est par contre mal prédite, quel que soit le code ou la densité du maillage. Ce problème est probablement lié à la mau- vaise description de la géométric de l'aube en son extrémité, telle qu'évoquée plus haut. Il peut également être dû à un calcul imprécis du développement de la couche-limite sur le profil. La comparaison de la vitesse axiale calculée et mesurée dans le sillage immédiat du profil à mi-envergure montre que cette couche-limite sur le profil est effectivement assez mal simulée, quel que soit le code ou la densité du maillage utilisé.

L'importance du maillage est illustrée par la comparaison des résultats de deux codes Navier-Stokes et par les résultats obtenus pour les différentes densités de maille du cas test. Au vu des ces résultats, on peut fixer comme limite inférieure pour le nombre d'éléments à disposer sur le rayon du cour du tourbillon une valeur intermédiaire entre les densités les plus faibles du cas test, c'est-à-dire un nombre d'éléments compris entre 4 et 10. Cette densité, qui peut paraître faible en absolu, est énorme lorsqu'il s'agit de prédire le développement d'un tourbillon dont la trajectoire est a priori inconnue et dont la taille du cœur est de l'ordre d'un centième de la corde du profil à son emplanture. Cela implique une taille moyenne des éléments, dans la zone probable de la propagation du tourbillon, de l'ordre d'un millième de la corde du profil. Dans le cas du calcul de l'aile elliptique, cette densité est tout juste atteinte pour le maillage raffiné 
(200 000 næuds) à la station $\mathrm{x} / \mathrm{C}=0,125$, mais pas pour les autres stations. Pour tous les autres maillages, ce nombre se situe au mieux à cinq, ce qui explique en partie les faibles rapports entre les vitesses tangentielles maximum calculées et mesurées.

Pour tous les calculs présentés, la diffusion du tourbillon calculée est beaucoup trop importante. Pour la section $\mathrm{x} / \mathrm{C}=0,5$, la vitesse tangentielle maximum prédite pour le maillage le plus fin est deux fois moins importante que celle mesurée.

\subsection{Analyse du cas test}

Une grande influence du solveur et du modèle de turbulence a été observée. L'analyse du cas test montre que seule l'utilisation d'un schéma de type Picard et d'un modèle de turbulence $k-\varepsilon$, donne une solution raisonnable, tous les autres surestimant de façon manifeste la diffusion du tourbillon. Si pour le modèle de turbulence $k-\varepsilon$, l'influence du solveur sur la solution obtenue est très importante, cela n'est pas le cas pour un modèle plus simple tel que le modèle de longueur de mélange. En effet, comme le montre la Figure 8, les solutions obtenues soit avec le schéma de Picard (s. s.), soit à l'aide du schéma découplé (segr.), sont rigoureusement identiques.

Pour un même schéma d'intégration, le résultat obtenu est fortement influencé par le choix modèle de turbulence et de ses paramètres. Spécialement pour le schéma d'intégration découplé par blocs, il semble que les conditions imposées en entrée du domaine de calcul, par le truchement de l'intensité de la turbulence ou de son échelle caractéristique, soient déterminantes pour le résultat obtenu. On constate sur la Figure 10 et la Figure 11 que, tant pour le modèle $k-\varepsilon$ que pour le modèle de longueur de mélange, l'importance de la diffusion du cœur du tourbillon est due à ces conditions amont. Cette influence de l'intensité de la turbulence en amont du profil sur la diffusion du tourbillon a également été remarquée par l'expérience [2], mais elle est nettement surestimée par la modélisation. De manière générale, il semble qu'il faille éviter d'imposer une échelle de la turbulence amont supérieure à la taille du cœur du tourbillon si l'on désire obtenir une diffusion raisonnable de ce dernier.

La diffusion trop importante du tourbillon peut être également liée au fait que l'on impose une condition soit de pression, soit de contrainte nulle en sortie du domaine de calcul. Cela peut avoir comme conséquence d'annihiler le tourbillon en sortie. Cette condition peut se reporter loin en amont dans le domaine de calcul, ce qui expliquerait cette diffusion trop importante. Cette raison, évoquée par certains auteurs [4], [6], n'a pas été mise en évidence par le cas test. Les résultats obtenus sur ce dernier semblent plutôt indiquer une influence du schéma d'intégration utilisé. Il est également probable que l'influence des conditions de sortie soie différente pour une approche volumes finis ou éléments finis. Dans le premier cas, seule la pression est imposée constante ou nulle, tandis que dans le deuxième cas, ce sont les contraintes qui sont annulées en sortie.

\section{V $\square$ CONCLUSIONS}

La capacité de trois codes de calcul à prendre en compte l'écoulement lié au développement d'un tourbillon marginal a été analysée. L'écoulement autour d'une aube de forme en plan elliptique et de section NACA 16020 placée dans une veine d'essai de section carrée a été calculé. Le champ de vitesse calculé est comparé, dans le sillage proche de l'aube et en son extrémité, à des mesures effectuées en diverses sections par vélocimétrie laser-Doppler. Afin de vérifier l'importance des paramètres du calcul mis en évidence par cette comparaison, un cas simplifié, correspondant à la propagation d'un tourbillon d'Oseen dans un tube, a été abordé.

Quelques conclusions peuvent être tirées de l'analyse de ces deux cas de calcul :

- Dans tous les cas, la diffusion du tourbillon prédite par le calcul s'est avérée beaucoup trop importante.

- Une grande influence tant du solveur que du modèle de turbulence sur le résultat obtenu a été mise en évidence. En particulier, on a montré qu'une longueur caractéristique de la turbulence amont inférieure au cœur du tourbillon marginal est nécessaire pour éviter une diffusion trop importante de ce dernier.

— Une densité du maillage d'au moins dix mailles dans le cœur du tourbillon est nécessaire pour obtenir un résultat satisfaisant. Cette condition impose des maillages très importants, puisque pour le maillage le plus dense présenté ici (200 000 noeuds), elle n'est atteinte que dans une très faible zone proche du l'extrémité du profil.

Ces conclusions montrent que la prédiction par le calcul des niveaux de pression associés à l'enroulement en extrémité d'un profil d'envergure finie n'est pas encore possible avec une grande précision. De ce fait, la prédiction numérique de l'apparition de la cavitation reste encore délicate.

Le problème de la densité du maillage pourra être résolu dans un avenir proche avec l'avènement de codes de calcul offrant la possibilité d'un maillage adaptatif. Celui du choix ou du développement d'un modèle de turbulence capable de prédire avec une bonne précision à la fois l'état de la couche-limite sur un profil à forte courbure et la mise en rotation de l'écoulement liée à l'enroulement du tourbillon est certainement beaucoup plus difficile.

\section{REMERCIEMENTS}

Cette étude a été réalisée dans le cadre de l'Action Concertée Cavitation grâce au soutien de la Direction des Recherches, Etudes et Techniques, Ministère de la Défense. Les auteurs tiennent également à témoigner leur gratitude à Messieurs $\mathrm{Ph}$. Cerrutti, Th. Pichon et J. Da Cunha qui ont réalisé les mesures vélocimétriques laser-Doppler présentées dans cet article.

\section{BIBLIOGRAPHIE}

[1] D. H. Fruman, P Cerruttr, T. Pichon, P. Dupont (mars 1995), - Effect of Hydrofoil Planform on Tip Vortex Roll-Up and Cavitation. Journal of Fluids Engineering, Vol. 117, pp. 162-169.

[2] A. Pauchet, X. Piot, D.H. Fruman (1996). - Effect of upstream Turbulence on Tip Vortex Roll-Up and Cavitation. Proceedings of the Cavitation and Multiphase Flow Forum. 1996 ASME Fluids Engineering Division Summer Meeting, San Diego, USA, Juillet 7-11.

[3] M.A.R. SHARIF, YKE. WONG (1995), - Evaluation of the Performance of Three Turbulence Closure Models in the Prediction of Confined Swirling Flows. Computers \& Fluids, Vol. 24, No. 1, pp. $81 \div 100$.

[4] J. Dacles-Mariani, G. G. Ziluiac, J. S. Chow, P. Bradshaw (1995). Numerical/Experimental Study of a Wingtip Vortex in the Near Field. AIAA Journal, Vol. 33, No. 9, septembre.

[5] Ph. Dupont, Ph. Cerrutl (1992), - Comparison between Tip Vortex Development Calculations and Measurements on an Elliptic Hydrofoil. Proceedings of 3rd European FIDAP Users Group Meeting, Heidelberg, Allemagne.

[6] F. DENISET (1996). - Modélisation numérique des conditions d'apparition de la cavitation de tourbillon marginal sur une aile 3-D. Effet de confinement. Thèse INPG. 